\title{
Vertical Load Uncertainty at the T/D Boundary under different spatial DER allocation techniques
}

\author{
Fabian Heymann, João Silva \\ CPES/Engineering faculty \\ INESC TEC/University of Porto \\ Porto, Portugal \\ fabian.heymann@fe.up.pt, \\ ipvs@inesctec.pt \\ Pablo Duenas \\ MITEI \\ Massachusetts Institute of Technology \\ Cambridge (MA), USA \\ pduenas@mit.edu
}

\author{
Philipe Vilaca \\ CPES \\ INESC TEC \\ Porto, Portugal \\ phillipe.v.gomes@inesctec.pt \\ Joel Melo \\ Federal University of $\mathrm{ABC}$ \\ CECS \\ Santo André, Brazil \\ joel.melo@ufabc.edu.br
}

\author{
Filipe Joel Soares \\ CPES \\ INESC TEC \\ Porto, Portugal \\ fsoares@inesctec.pt \\ Vladimiro Miranda \\ CPES/Engineering faculty \\ INESC TEC/University of Porto \\ Porto, Portugal \\ vmiranda@inesctec.pt
}

\begin{abstract}
Vertical load is the power flow between electrical transmission and distribution networks. In the past, large-scale generators connected to transmission systems supplied consumers connected to lower voltage levels across distribution grids. Thus, vertical loads tended to be downward-oriented. This paper presents a spatiotemporal distributed energy resources (DER) diffusion model to analyze vertical load uncertainty resulting from different DER diffusion process representations currently used in the industry and academia. Network planners and operators can use such model to understand the long-term evolution of load at the T/D boundary. The proposal is applied to the Portuguese power system, combining, as first of its kind, highly granulated population census with georeferenced transmission and distribution network datasets. This application analyzes the 20-year evolution of such vertical load flows at the transmissiondistribution boundary under a strong uptake of DER embodied in lower voltage levels in Portugal.
\end{abstract}

Keywords-Diffusion of Innovations Theory, Distributed Energy Resources, Transmission system planning, Spatiotemporal modeling.

\section{INTRODUCTION}

\section{A. Background}

The transformation towards low-carbon, decentralized power systems go hand-in-hand with a rapid uptake of new distributed energy resources (DER) that are increasingly connected to electricity distribution networks. Consecutively substituting centralized generation that used to be connected to the higher voltage levels (transmission) with low-/medium voltage installations, a large-scale adoption of DER is about to affect the vertical load exchange between transmission and distribution (T/D) networks [1].

As many power systems recently implemented unbundling policies, there is no institutional connection of transmission and distribution system planning anymore. The resulting stop of information flows as well as the spatial stochasticity of DER adoption patterns call for actualized methods that can frame the growing uncertainty in T/D grid planning [2].

The authors gratefully acknowledge the provision of data-sets by the Portuguese Energy Agency (ADENE) and CEiiA as well as the financial support granted under FCT-MIT Portugal Scholarship PD/BD/114262/2016 and INCT-INERGE, CNPq (grant: 422044/2018$0)$ and Sao Paulo Research Foundation (FAPESP) under grant: \#2017/22577-9, \#2019/00466-6.
This work presents the spatial methodology to characterize the uncertainty in load flows between the transmission and distribution boundary due to the DER diffusion, considering a high level of spatial and temporal granularity. Such load flows have been called vertical load, equivalent to the "the sum, positive or negative, of all power transferred from the transmission grid through directly connected transformers and power lines to distribution grids and final consumers" [3].

The application of the proposed spatiotemporal DER diffusion model relies on highly granular census data (over 17,000 census cells for Continental Portugal) and is used to forecast EV and PV adoption on a 20-year horizon. Model outcomes are net-load curves for each of the distribution network's HV/MV transformer service areas. From these outcomes and using the information on the physical connection of each $\mathrm{HV} / \mathrm{MV}$ substation to a transmission system entry point, the vertical load diagram between the distribution and transmission interface can be computed. In addition to research presented in [4], the results of four different DER allocation techniques are compared.

\section{B. Research Gap and Article contributions}

The article contributes to the emerging body of literature dedicated to the analysis of future, coordinated transmissiondistribution network operation and planning through:

- An extensive review of transmissiondistribution (T/D) cooperation schemes and network expansion planning under DER diffusion.

- The application of a spatiotemporal DER diffusion model to electricity network planning.

- The establishment of two new planning criteria that describe the system's net-load behavior at the T/D interface (total reverse flow hours and total peak load addition).

- A comparison of results retrieved across four typical DER allocation techniques commonly applied for grid impact studies.

Eventually, the presented model will serve distribution and transmission network planners to downscale global DER diffusion forecasts and assess the impact of technology diffusion dynamics on network planning. While comparing the outcomes of four different DER allocation techniques, one can obtain an improved understanding of the uncertainty in case only one DER allocation technique used to be applied. 
Table I. DER allocation methods in distribution networks [4]

\begin{tabular}{ll} 
Type & DER Allocation technique \\
\hline Deterministic & - Extrapolation using busbar capacities \\
& - Extrapolation using peak demand \\
Randomized & - Single-step random allocation \\
& (e.g. equal assignment) \\
& - Multi-step iterative allocation \\
& (e.g. using Monte Carlo)
\end{tabular}

\section{LITERATURE REVIEW}

\section{A. Representation of DER diffusion in network studies}

With the rise of DER adoption in power systems, an increasing amount of research has been dedicated assessing the impact of such technologies to distribution or transmission networks. However, little evidence has been accumulated on how different ways of representing DER uptake in transmission or distribution networks affect network expansion and investment forecasts. As shown in [4], strong differences in such outcomes can be achieved under the currently most utilized DER allocation techniques.

Typically, current techniques do not consider consumer/population distributions within the test networks analyzed. Popular approaches are shown below (Table I) and include equal or random assignment of DER across all busbars, or extrapolation on installed busbar capacities or demand peaks. In this paper, we analyze the effects of different allocation techniques on load diagram results that describe the vertical flows between the distribution and transmission system in Continental Portugal.

\section{B. Spatial net-load forecasting in transmission planning}

New trends such as the uptake of DER can change consumer habits and thus, peak-load behavior. Hence, planners must analyze, in a spatial and temporal form, consumer locations that might develop consumption habit changes under DER adoption [5].

The spatial prediction of electricity demand and its change over time is the domain of spatial load forecasting (SLF). A detailed review of SLF and recent developments can be found in [6]. In the past, studies of spatial load forecasting at the level of power transmission networks had little use as stochastic factors of the electric demand were less influential over the planning horizon in these studies [7]. However, due to the high concentration of DER in some regions of the electrical power system especially in the first years of DER technologies diffusion, the simplistic load growth scenarios previously considered need to be refined [8].

The results of spatial load forecasting at the level of electrical distribution systems can provide useful information for the study of load growth that must be met by the transmission networks. In the literature, first works that introduced higher spatial granularity to transmission planning [9], an introduction of the spatially resolved vertical load concept [10] and DER diffusion forecasting models [8] have been presented. However, an approach that displayed how to integrate all mentioned aspects has been absent so far.

\section{Vertical flows at the Transmission/Distribution interface}

The increasing penetration of DER across distribution grids and its impact on the vertical load has become an operational and planning challenge for both distribution and transmission system operators (DSO and TSO). Technical problems (e.g., voltage problems, branch congestion) will occur more frequently in both networks due to the arising of reverse power flows. Ensuring the quality of service and security of supply will thus be a hard task for network operators that need to account for the uncertainty associated to these resources and the lack of T/D power flow coordination.

Traditionally, the TSO carries the main responsibility for ensuring overall system security. Therefore, the existing coordination mechanisms between her and the DSO regarding most grid operation challenges (e.g. congestion management, voltage support) are scarce [11], [12]. However, the paradigm change associated with an "active" distribution grid is a clear sign that cooperation procedures such as the estimation of load vertical uncertainty are of utmost importance [13].

In [14], an attempt to estimate the active and reactive power vertical flow limits has been presented. The work considered a real French distribution test network and the results highlighted the capability of these types of studies.

Despite previous efforts, the impact analysis of DER uncertainty on these vertical loads has not yet been studied. This paper focuses exactly on this aspect and the final conclusions might be very useful for studies like the one described in [14]

\section{Transmission expansion under DER adoption}

As DER adoption has the potential to meet the distribution system load, the transmission network may become less congested depending on the level of the adopted local capacity. For these reasons, studies on transmission planning under DER adoption have been developed over the last years. The work in [15] presents an approach to choose an optimal portfolio of DER services that allows to reduce required transmission expansion investments. The results of this approach prove the benefits of security services provided by DER and their advantages in postpone transmission investment.

The modifications of the load patterns caused by distributed generation and anticipated flexibility additions at the transmission-distribution boundary have been presented in [16]. Results indicate that the penetration of distributed generation (PV) can provide added value to system through the reduction of operation costs, transmission losses and $\mathrm{CO}_{2}$ emissions. Nevertheless, PV systems may not, on an isolated way, contribute to alleviate the demand seen by transmission networks in peak load periods (where TEP is generally conducted). On the other hand, growing electrification of the transport sector is posing additional complexity to transmission planning. Here, first research outcomes showed that a concerted use of EV charging policies can constrain transmission expansion investments [17]. 


\section{METHODOLOGY}

\section{A. A spatial model of the $T / D$ interface}

One innovation of this paper is the computation of transmission service areas, using $\mathrm{HV} / \mathrm{MV}$ transformer positions (a) and tabular information of the linkage of those to a certain transmission entry point (Fig. I). Approximating HV/MV transformer service areas as a first step (b) through a Voronoi diagram as in [18], the retrieved spatial polygons are further merged (c) based on the information of their connection to each transmission entry point, available in [19]. The resulting polygons (c) are consecutively linked with the census data-set through spatial operations (overlaying). That way, each transmission entry service area is linked to detailed information of the population subgroup alimented by each specific transmission entry node.

\section{B. Spatiotemporal DER diffusion model}

The presented approach builds on a 6-step spatiotemporal model, described in [4]. The model builds on census data with high spatial granularity and global technology adoption scenarios and simulates spatial technology adoption patterns. In this article, the model is used to estimate the impact of EV/PV adoption onto vertical load diagrams, that is the load flow between distribution and transmission network.

The load diagrams are computed for each of the 63 entry points to the transmission network. The extensive discussion of the technology diffusion model is outside the scope of this paper. An interested reader may find a more detailed explanation in [4] and its application to case studies in [20]. Its 6 subroutines are described below:

i. Geolocation routine that relates census polygons with varying spatial extent and population characteristics to HV/MV service areas.

ii. Spatial routine that computes the HV/MV substation service areas around transformer locations (XY coordinates) using Voronoi diagram.

iii. HV/MV transformer service area aggregation and capacity estimation at each T/D connection point.

iv. A diffusion pattern generator that forecasts EV and PV adopter locations using census cells as input.

v. A net-load analysis that considers hourly load, EV charging and PV generation time series.

vi. Vertical load flow analysis routine that uses the hourly net-load time $(\boldsymbol{N L})$ series $(8760 \mathrm{~h})$.

\section{Vertical load diagram analysis}

This paper contains a thorough analysis of various largescale DER allocation techniques (at) and their resulting vertical load flow estimates. The presented model is eventually used to quantify a range of indicators that characterize the load flows at the T/D boundary of the continental Portuguese power system.

As in [4], the netload for each HV/MV service area (sa) and each hour $(\boldsymbol{h})$ is calculated, subtracting a PV curve $(\boldsymbol{P V})$ from the addition of load $(\boldsymbol{L})$ and EV charging $(\boldsymbol{E} \boldsymbol{V})$.

$$
N L_{s a, h}=L_{s a, h}+E V_{s a, h}-P V_{s a, h}
$$

a)
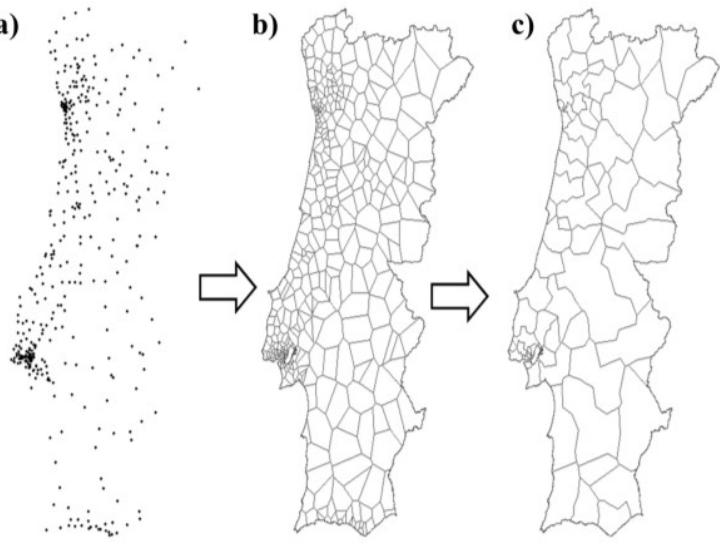

Figure I. Retrieval of spatial T/D network service areas

For each transmission service area, all $\boldsymbol{N L}$ values for each connected HV/MV substation is added. Peak-load coincidence behavior is neglected. The impact assessment includes time-series analysis on the changes of load diagrams, including the change in flow directions (distribution- $>$ transmission and distribution $<-$ transmission) and peak-load behavior at each T/D connection point. The following metrics are analyzed:

$$
R F_{a t}=\sum_{h=1}^{8760}\left\{\begin{array}{l}
1 \text { if } N L_{h}<0 \\
0 \text { if } N L_{h} \geq 0
\end{array}\right\}
$$

Here, RF is an hour where reverse flows are experienced in a given transmission service area. RF hours has parallels to the Loss of Load Expectation (LOLE) concept, summing the occurrences of specific situations while neglecting magnitude. $\boldsymbol{R} \boldsymbol{F}$ are calculated for each transmission service area as well as DER allocation technique.

A second indicator used in this work is the peak load added $(\boldsymbol{P} \boldsymbol{A})$ under each DER allocation technique for every transmission service area. In absence of any information of the transmission system configuration and components (no line or transformer loading calculations possible), this metric provides indication of the peak load added to the reference value (peak load in year 0 of the natural load).

$$
P A_{a l}=\sum_{s a=1}^{63}\left(P L_{\text {Year } 20}-P L_{\text {Year } 0}\right)
$$

The metric is calculated subtracting peak values after 20 years of EV/PV adoption from natural load peak values. Such differences are retrieved for each transmission service area (sa) and across all DER allocation techniques (at).

\section{INPUT DATA}

The presented model relies in four different data sources that are detailed below:

- Census dataset that originates from 2011 [21]. It contains a detailed description of the Portuguese population through a set of over 120 sociodemographic and building-related census variables (age, education, employment status, building type and apartment size). The census variables come with 
a spatial dataset that contains over 17,000 census tracts (neighborhoods).

- $\quad \mathrm{EV} / \mathrm{PV}$ adoption scenarios for 2035, respecting a 20 year planning horizon and 2015 as base year [22].

- Distribution network information such as HV/MV substation characterizations (location, peak load, installed capacity) and the linkage of substations to each of the 63 transmission entry points [19], [23].

- Typical natural load (assumed to incorporate already adopted EV/PV) MV, EV charging and PV generation time series. Respective time series have been normalized to peak values and are stretched to the forecasted EV/PV capacities at each HV/MV transformer (per capita PV potential was estimated as $0.4 \mathrm{kWpeak} /$ capita; EV adopter charge at a rate of $5.9 \mathrm{~kW}$ under a 0.5 simultaneity rate).

The global EV/PV forecast was derived using the Bass model [24]. Chosen coefficients are shown below (Table II). As this report aggregates both large-scale and dispersed PV installations in residencies, the forecast was reduced using the current ratio of dispersed $\mathrm{PV}$ to overall $\mathrm{PV}$ installations in Portugal as stated in [25]. The calculation of the total EV and
Table II. Bass model parameters of the proposed methodology

\begin{tabular}{|l|c|c|c|}
\hline Technology & $\mathrm{p}$ & $\mathrm{q}$ & $\mathrm{m}$ \\
\hline Electric vehicles & 0.000618 & 0.873600 & $1,305,055$ \\
\hline Photovoltaics & 0.000618 & 0.873600 & $3,759,570$ \\
\hline
\end{tabular}

PV potentials $(\boldsymbol{m})$ at all residencies were based on [26] and [4]. The model coefficients $\boldsymbol{p}$ and $\boldsymbol{q}$ have been retrieved, calibrating the model with historical uptake values.

\section{RESULTS}

Results reveal the large differences in EV and PV distributions within transmission service areas that result from the use of different DER allocation techniques. Looking at the maximum deviation for all previously introduced algorithms, predicted EV charging power forecasts might differ by magnitudes of up to $48 \mathrm{MW}$ per service area, whereas residential PV installation forecasts may vary even up to $97 \mathrm{MW}$ per service area. Minimum, maximum and range of both EV charging and PV installation forecasts are shown in Figure II and Figure III respectively.

This underlines the sensitivity of transmission and distribution network planning to the type of DER diffusion model (or, allocation technique) that is used once a global
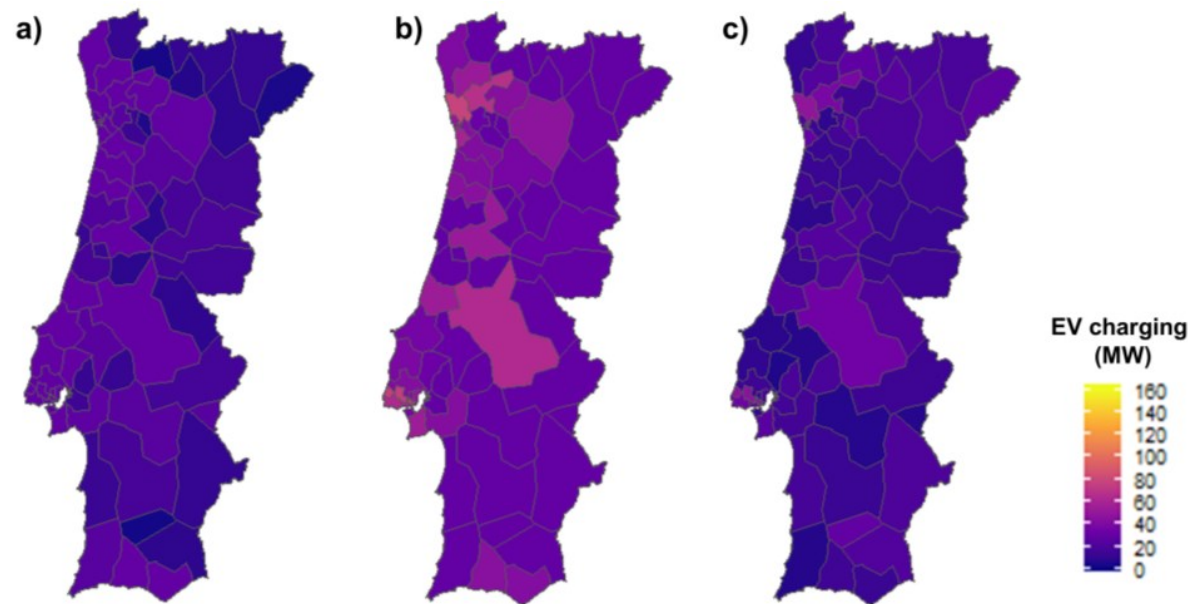

Figure II. EV diffusion for different EV allocation techniques, where a) max, b) min and c) maximum difference

a)

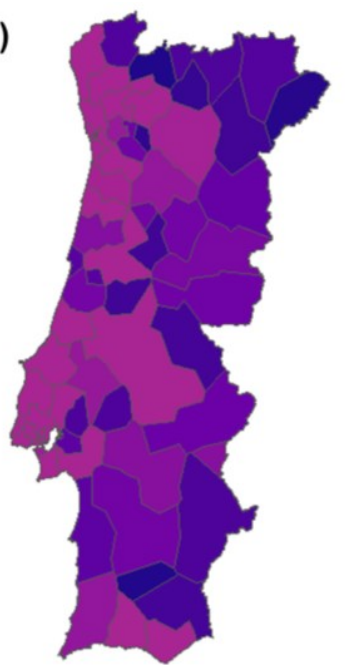

b)

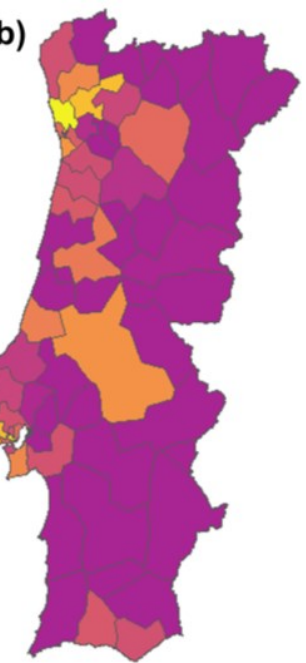

c)

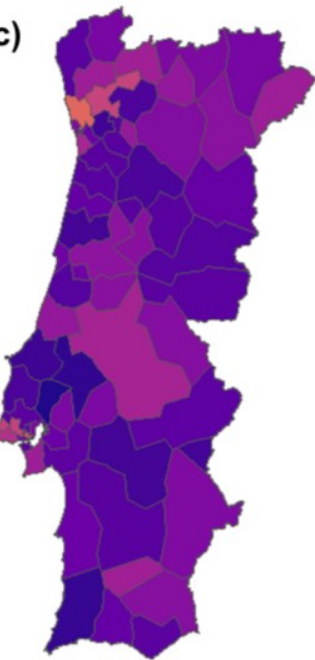

PV installed

(MW)

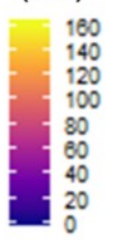

Figure III. PV diffusion for different PV allocation techniques, where a) max, b) min and c) maximum difference 
forecast is downscaled. Such effects are demonstrated in the figures below.

Figure IV displays load curves for two transmission service areas that exhibit either the maximum amount of reverse flow hours (a) and maximum addition to its existing peak load value (b). Situation a) occurs in a small area in the North of Portugal - under an equal-share DER allocation. Here, given a low natural peak load (before DER adoption occurs), the application of the equal DER allocation technique leads to a strong stretching of the initial load curve (Figure IV.a) through added roughly $20 \mathrm{MW}$ EV charging. Likewise, such approach would allocate almost $60 \mathrm{MW}$ of $\mathrm{PV}$ installations to the respective transmission service area, resulting in frequent reverse flow estimates (Figure IV.a).

Situation b) locates in a populated southern zone inside Lisbon Metropolitan area and represents the case of maximum peak load addition under all DER allocation techniques and service areas considered. The respective DER forecast has been retrieved relying on a diffusion study approach that is sensitive to the population counts connected to each transmission service area. Results are shown both in Figures IV.b and V.b and validate the intuition that under conditions with high peak loads, reverse flows are less likely.
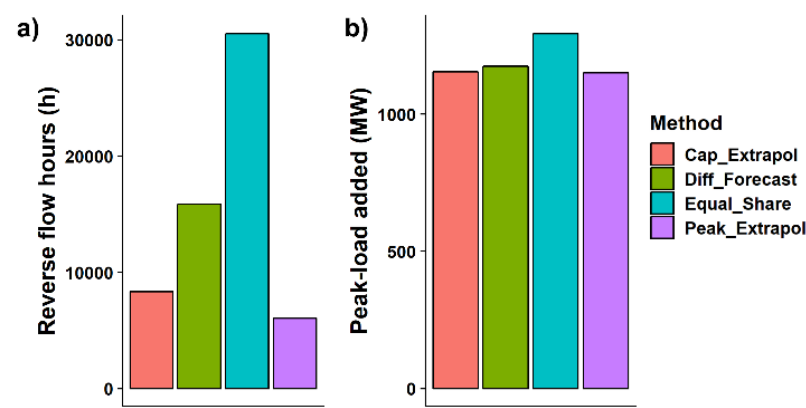

Figure VI. Estimated reverse flow hours (a) and peak-load increments (b) over the transmission system under different DER allocation techniques

Likewise, newly introduced, global indicators for the vertical load (the flow between transmission and distribution) have been derived (Figure VI). These include the annual expected amount of reverse flow hours (from distribution to transmission) and the total expected peak addition for each transmission service area. While latter are expected to grow similarly under all DER allocation techniques on a global perspective, reverse flow hour estimates vary strongly, between roughly 5,000 and 30,000 hours for all transmission service areas combined (Fig. VI).

a)

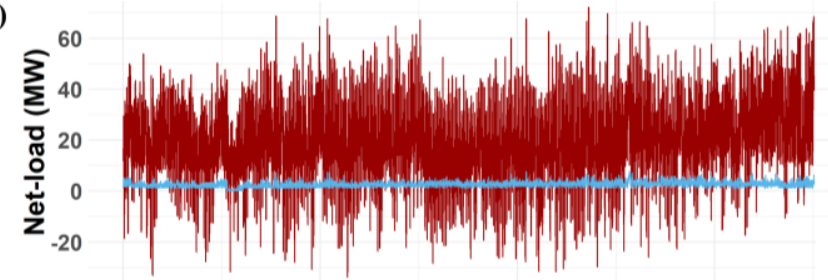

b)

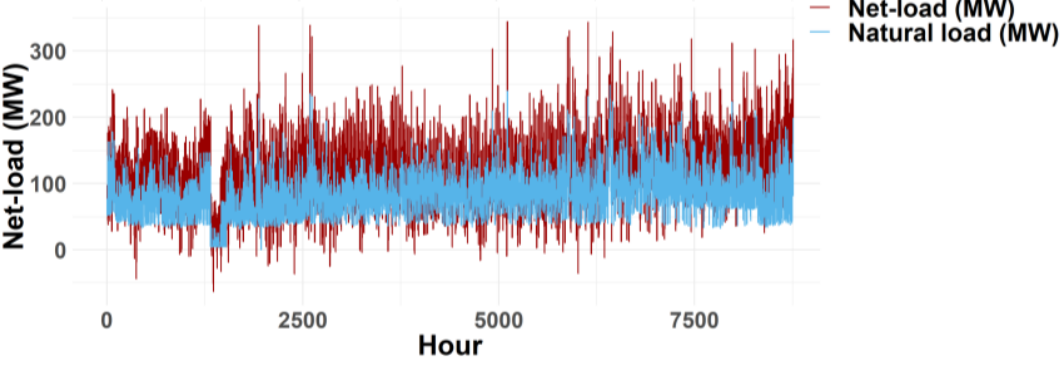

Figure IV. Load curves for transmission service area with most frequent reverse flows (a) and maximum peak increment (b)

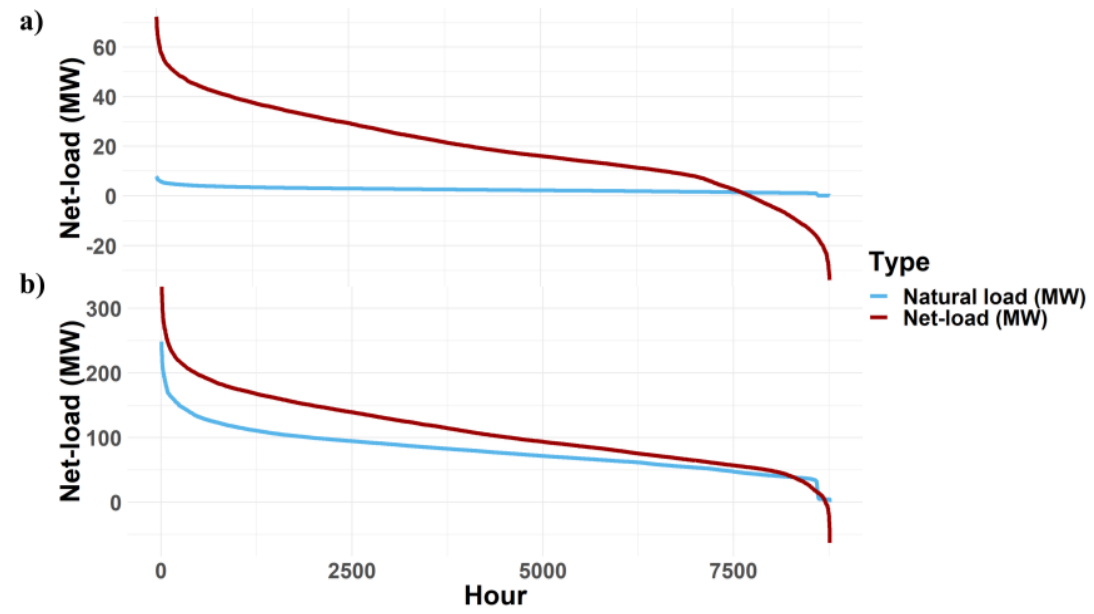

Figure V. Load duration curves for transmission service area with most frequent reverse flows (a) and maximum peak increment (b) 


\section{CONCLUSIONS AND FUTURE WORK}

The presented paper compared four commonly used techniques that model the uptake of DER in transmission and distribution networks. A global DER forecast on a 20 -year horizon is used to unveil the differences that can occur if one or another technique is used.

As a first innovation, the presented paper estimates the spatial extent of transmission service areas, based on HV/MV transformer locations and their connectivity to transmission entry points only. Results show that the choice of DER allocation algorithm can heavily affect load diagrams retrieved. For example, regional deviations of up to $48 \mathrm{MW}$ residential EV charging and even $98 \mathrm{MW}$ household-based PV installations could be observed across the transmission system service areas. Likewise, newly introduced, global indicators for the vertical load (the flow between transmission and distribution) have been derived.

These include the annual expected amount of reverse flow hours (from distribution to transmission) and the total expected peak addition for each transmission service area. While latter both are expected to grow similarly under all DER allocation techniques on a global perspective, reverse flow hour estimates vary strongly, between roughly 5,000 and 30,000 hours for all transmission service areas combined.

As a principal take-away, outcomes call for improved granularity and careful calibration of DER forecast to enhance transmission expansion and investment decisions.

\section{REFERENCES}

S. Lumbreras and A. Ramos, "The new challenges to transmission expansion planning. Survey of recent practice and literature review," Electr. Power Syst. Res., vol. 134, pp. 19-29, 2016.

K. Bell, "Methods and Tools for Planning the Future Power System: Issues and Priorities," Model. Requir. GB Power Syst. Resil. Dur. Transit. to Low Carbon Energy, no. Paper 5, pp. 1-35, 2015.

[3] Amprion, "Vertical load," 2018. [Online]. Available: https://www.amprion.net/Grid-Data/Vertical-Load/. [Accessed: 12-Nov-2018].

[4] F. Heymann, J. Silva, V. Miranda, J. Melo, F. J. Soares, and A. Padilha-Feltrin, "Distribution network planning considering technology diffusion dynamics and spatial net-load behavior," Int. J. Electr. Power Energy Syst., vol. 106, pp. 254-265, 2019.

[5] A. P. Robinson, P. T. Blythe, M. C. Bell, Y. Hübner, and G. A. Hill, "Analysis of Electric Vehicle Driver Charging Behaviour and Use of Charging Infrastructure," 18th World Congr. Intell. Transp. Syst., no. January, 2012.

[6] F. Heymann, J. Melo, P. D. Martínez, F. Soares, and V. Miranda "On the Emerging Role of Spatial Load Forecasting in Transmission / Distribution Grid Planning," in 11th Mediterranean Conference on Power Generation, Transmission, Distribution and Energy Conversion (MEDPOWER 2018), 2018.

[7] J. R. Aguero and H. L. Willis, "Spatial Electric Load Forecasting Methods for Electric Utilities," Raleigh, 2007.

[8] A. D. Mills, "Forecasting load on the distribution and transmission system with distributed energy resources," in Distribution Systems and Planning Training for Midwest Public Utility Commissions,
2018, pp. 1-38.

[9] S. Fan, K. Methaprayoon, and W. J. Lee, "Multiregion load forecasting for system with large geographical area," IEEE Trans. Ind. Appl., vol. 45, no. 4, pp. 1452-1459, 2009.

[10] M. Robinius, F. ter Stein, A. Schwane, and D. Stolten, "A TopDown Spatially Resolved Electrical Load Model,” Energies, vol. 10, no. 3, p. 361, 2017.

[11] A. Zegers and H. Brunner, "TSO-DSO interaction: An Overview of current interaction between transmission and distribution system operators and an assessment of their cooperation in Smart Grids," ISGAN Discuss. Pap. Annex 6 Power T\&D Syst. Task 5, no. September, 2014.

[12] H. Gerard, E. Rivero, and D. Six, "Basic schemes for TSO-DSO coordination and ancillary services provision," 2016.

[13] ENTSO-E, CEDEC, GEODE, EURELECTRIC, and EDSO, “TSO-DSO Data Management Report," 2015.

[14] M. Caujolle et al., "Estimating the Active and Reactive Power Flexibility Area at the TSO-DSO Interface," IEEE Trans. Power Syst., vol. 33, no. 5, pp. 4741-4750, 2018.

[15] D. Alvarado, A. Moreira, R. Moreno, and G. Strbac, "Transmission Network Investment with Distributed Energy Resources and Distributionally Robust Security," IEEE Trans. Power Syst., vol. PP, no. c, p. 1, 2018.

[16] P. V. Gomes and J. T. Saraiva, "Transmission system planning considering solar distributed generation penetration," Int. Conf. Eur. Energy Mark. EEM, pp. 2-7, 2017.

[17] P. V. Gomes, J. T. Saraiva, M. D. P. Coelho, B. H. Dias, L. Willer, and A. C. Junior, "Impact of large fleets of plug-in-electric vehicles on transmission systems expansion planning," 20th Power Syst. Comput. Conf. PSCC 2018, pp. 1-7, 2018.

[18] A. Navarro and H. Rudnick, "Large Scale Distribution Planning Part II : Macro-optimization with Voronoi's Diagram and Tabu Search," IEEE Trans. Power Syst., vol. 24, no. 2, pp. 752-758, 2009.

[19] EDP Distribuição, "Distribution Network Development and Investment Plan 2017-2021 (in Portuguese)," 2016.

[20] F. Heymann, N. Neyestani, F. J. Soares, and V. Miranda, "Mapping the Impact of Daytime and Overnight Electric Vehicle Charging on Distribution Grids," in 2017 IEEE Vehicle Power and Propulsion Conference (VPPC), 2017.

[21] INE, “Import of georeferenzed Census 2011 data (BGRI)," 2011. [Online]. Available: http://mapas.ine.pt/download/index2011.phtml. [Accessed: 01Jan-2017].

[22] ENTSO-E, “TYNDP 2018: Scenario Report,” 2018.

[23] EDP Distribuição, "National distribution grid - High Voltage and substations," 2016. [Online]. Available: http://edp-distribuicaorede.wntech.com/Nut/4. [Accessed: 05-Sep-2017]

[24] F. M. Bass, "A New Product Growth for Model Consumer Durables," Manage. Sci., vol. 5, no. 16, pp. 215-227, 1969.

[25] Portuguese General Direction of Energy and Geology (DGEG), "Rapid Statistics - Renewables No.168," 2018.

[26] ACAP, "Estatísticas do Sector Automóvel," Assoc. Automóvel Port., 2013. 